\title{
Cuantificación de V, Ni, Zn y Fe en asfalto por espectroscopia de absorción atómica.
}

\section{QUANTIFICATION OF V, Ni, Zn and Fe IN ASPHALT BY ATOMIC ABSORPTION SPECTROSCOPY.}

\section{Lic. Jocseline M. Hidalgo Porras}

Estudiante Laboratorista Químico, Universidad de Costa Rica,

Costa Rica

jhidalgopor@gmail.com

Fecha de recepción: 24 AGOSTO 2017 / Fecha de aprobación: 27 NOVIEMBRE 2017.

Índices y Bases de Datos:

\section{lationdex ucRIndex}

\section{REDIB O Dialnet DOA}

revistas.ucr.ac.cr/index.php/materiales

(c) lanamme.ucr.ac.cr

@ metodosymateriales.lanamme@ucr.ac.cr
Políticas de Uso:

\section{(c) $\underset{\mathrm{BY}}{(1)(9)}$}

Revista Métodos y Materiales por LanammeUCR se distribuye bajo: Licencia Creative Commons Atribución-NoComercial-SinDerivar 4.0 Internacional. ISSN impreso: 2215-342X. ISSN electrónico: 2215-4558 


\title{
Cuantificación de V, Ni, Zn y Fe en asfalto por espectroscopia de absorción atómica.
}

\section{QUANTIFICATION OF V, Ni, Zn and Fe IN ASPHALT BY ATOMIC ABSORPTION SPECTROSCOPY.}

\author{
Lic. Jocseline M. Hidalgo Porras \\ Estudiante Laboratorista Químico, Universidad de Costa Rica, \\ Costa Rica \\ jhidalgopor@gmail.com
}

Fecha de recepción: 24 AGOSTO 2017 / Fecha de aprobación: 27 NOVIEMBRE 2017.

\section{RESUMEN}

El asfalto tiene complejas composiciones químicas y físicas que normalmente varían con la fuente del petróleo crudo. Su composición se basa en aproximadamente $84 \% \mathrm{C}, 10 \% \mathrm{H}_{2}, 1 \% \mathrm{O}_{2}$ y $5 \%$ trazas de elementos como $\mathrm{S}, \mathrm{Ni}, \mathrm{V}$ y $\mathrm{Fe}$. Se trata del desarrollo de un nuevo método para cuantificar V, Ni, Zn y Fe en asfalto AC-30 por espectroscopia de absorción atómica. Estos metales fueron detectados cualitativamente con una mayor intensidad en un análisis de Rayos $\mathrm{X}$ por fluorescencia en un microscopio de barrido de electrones. Algunos metales encontrados en el asfalto pueden tener un efecto catalítico en la oxidación del asfalto. La investigación propone un procedimiento de tratamiento de hasta $2 \mathrm{~g}$ de asfalto, el cual se basa en su dilución con solvente orgánico (mineral spirits) en balones aforados con ayuda de un baño ultrasónico para aligerar la dilución. Se dictan los parámetros de optimización del método buscando los datos instrumentales adecuados en el equipo de absorción atómica para la obtención de la mayor absorbancia posible en la alineación tanto del quemador como de las lámparas de cátodo hueco y deuterio; además de la optimización de la llama y del flujo del nebulizador. Las curvas de calibración de cada metal se realizaron con un estándar con base de hidrocarburos para medir las concentraciones de metales en el asfalto en $\mathrm{mg} / \mathrm{kg}$ con un coeficiente de correlación lineal de mínimo 0,995. El estudio de recuperación en asfalto se realiza con la adición de una cantidad de los metales directamente a las alícuotas de matriz de asfalto. Las concentraciones obtenidas para el Ni fueron 70, V 330, Zn 24 y para Fe $10 \mathrm{ppm}$, los cuales presentaron una desviación estándar relativa inferiores al $1 \%$ lo que indica que las mediciones de una misma muestra son precisas y que presentan poca dispersión entre ellas. Las recuperaciones promedio fueron de $99,17 \%$ para $\mathrm{Ni}, 100,30 \%$ V, 92,26\% Zn y $97,72 \%$ Fe. Estos resultados indican que el método resulta confiable para la cuantificación de metales mediante la técnica de absorción atómica; valores superiores al $100 \%$ se dan por lecturas de absorbancias superiores al esperado o por generación de falsos positivos en la técnica.

PALABRAS CLAVE: Asfalto, porfirinas, absorción atómica, metales, espectroscopia.

\section{ABSTRACT}

Asphalt has complex chemical and physical compositions that normally vary with the source of crude oil. Its composition is based on approximately $84 \% \mathrm{C}, 10 \% \mathrm{H}_{2^{\prime}} 1 \% \mathrm{O}_{2}$ and $5 \%$ trace elements such as S, Ni, $\mathrm{V}$ and Fe. This is the development of a new method to quantify $\mathrm{V}, \mathrm{Ni}, \mathrm{Zn}$ and Fe on asphalt AC-30 by atomic absorption spectroscopy. These metals were detected qualitatively with a higher intensity in an X-ray analysis by fluorescence in an electron scanning microscope. Some metals found in asphalt may have a catalytic effect on the oxidation of asphalt.The research proposes a treatment process of up to $2 \mathrm{~g}$ of asphalt, which is based on dilution with mineral spirits in volumetric flasks with the aid of an ultrasonic bath to lighten the dilution. The parameters of optimization of the method are dictated by looking for the adequate instrumental data in the atomic absorption equipment to obtain the highest possible absorbance in the alignment of both the burner and the hollow cathode and deuterium lamps; In addition to the optimization of the flame and the flow of the nebulizer. The calibration curves of each metal were performed with a hydrocarbon-based standard to measure metal concentrations in asphalt in $\mathrm{mg} / \mathrm{kg}$ with a linear correlation coefficient of at least 0,995. The asphalt recovery study is performed with the addition of a quantity of the metals directly to the asphalt matrix aliquots. The concentrations obtained for Ni were 70, V 330, Zn 24 and for Fe 10 ppm, which presented a relative standard deviation of less than $1 \%$, indicating that measurements of the same sample are accurate and have little dispersion between them. The average recoveries were $99,17 \%$ for $\mathrm{Ni}, 100,30 \% \mathrm{~V}, 92,26 \% \mathrm{Zn}$ and $97,72 \%$ $\mathrm{Fe}$. These results indicate that the method is reliable for the quantification of metals by the atomic absorption technique; values greater than $100 \%$ are given by readings of absorbances higher than expected or by generation of false positives in the technique.

KEY WORDS: Asphalt, porphyrins, atomic absorption, metals, spectroscopy. 


\section{INTRODUCCIÓN}

El asfalto tiene complejas composiciones químicas y físicas que normalmente varían con la fuente del petróleo crudo y se considera como dispersiones de partículas (asfaltenos) en un fluido de alto punto de ebullición formado por aceite y resina (Speight, 2014). Las grandes moléculas de asfaltenos están rodeados por compuestos aromáticos polares y no polares, suspendidas en aceites saturados, siendo los responsables del comportamiento viscoelástico del asfalto a temperatura ambiente (Cárdenas y Fonseca, 2009).

La masa principal de asfalto es un hidrocarburo que presenta una estructura química primaria que incluye anillos aromáticos, anillos cíclicos (aromáticos nafténicos), alifáticos (alcanos), cuyas moléculas contienen heteroátomos $(\mathrm{O}, \mathrm{N}$, S) que forman asociación entre moléculas, las piridinas (inducen polaridad) e influyen en forma significativa en el comportamiento mecánico del asfalto (Cárdenas y Fonseca, 2009). En menor proporción se encuentran en los asfaltos metales como el vanadio, hasta 2000 ppm y níquel hasta 200 ppm (Cortés, 2012), éstos se correlacionan con la cantidad de azufre y asfaltenos de los mismos bitúmenes formando porfirinas (Nahar et al., 2015). El contenido de Ni y V se correlaciona fuertemente con el peso molecular del asfalto y el índice de envejecimiento se correlaciona fuertemente con el contenido de Ni (Bishara, 1997).

El envejecimiento afecta las propiedades mecánicas del asfalto ya que se puede presentar por un lado un pavimento muy suave lo que provoca que se ahuelle y se deforme, o por otro lado un pavimento demasiado rígido y frágil, el cual está entonces sujeto a grietas, ya sea en virtud de la carga de tráfico o debido al bajo estrés térmico (Robertson et al., 1991). La oxidación del asfalto es uno de los efectos indeseables debido al deterioro de las propiedades físicas del asfalto, ya que provoca un endurecimiento del pavimento. Este endurecimiento por envejecimiento oxidativo contribuye significativamente a la fragilidad del pavimento, lo que conlleva a un agrietamiento del pavimento en forma excesiva (Speight, 2016).

Además del V, Ni y Fe que están presente a una concentración significativa en muchos bitúmenes, hay otros metales como $\mathrm{Mg}, \mathrm{Ca}, \mathrm{Cr}, \mathrm{Co}, \mathrm{Zn}$ y Mo que están con frecuencia en cantidades más pequeñas que influyen en las interacciones moleculares dentro del material. La concentración de estos elementos y su asociación varían dependiendo de la fuente del betún. El conocimiento de estos elementos puede proporcionar información útil sobre el origen de la fuente de petróleo crudo o puede proporcionar una huella digital única del origen de un betún (Nahar et al., 2015).
En Costa Rica el principal uso del asfalto se da en la construcción y en el mantenimiento y rehabilitación de las carreteras. Entre los análisis que se realizan para determinar las condiciones del estado del pavimento, no se han incluido aún estudios para relacionar la concentración de los metales contenidos en el asfalto con el rendimiento del mismo en el pavimento, ni cómo esta variable afecta la oxidación del mismo. Es probable que el nivel de concentración de los metales en ppm pueda relacionarse con el rendimiento del asfalto en el pavimento, ya que algunos metales podrían actuar como catalíticos del proceso de oxidación en el asfalto (Branthaver et al.,1984). La cuantificación de V, $\mathrm{Ni}, \mathrm{Zn}$ y Fe proporciona información de la abundancia de cada metal existente en el asfalto AC-30; información que posteriormente se puede relacionar con posibles reacciones redox o catalizadores de la oxidación, y así sustentar si hay participación de estos metales en la oxidación de los materiales bituminosos. Esto permitiría hacer mejoras en los ligantes asfálticos con el fin de aumentar la resistencia del asfalto original a los efectos perjudiciales del endurecimiento por envejecimiento oxidativo al mejorar su rendimiento, fortaleciendo la infraestructura civil y vial principalmente (García y Melgar, 2011).

La caracterización química del asfalto ha sido desarrollada mediante diversos sistemas analíticos, la espectroscopia de absorción atómica en química analítica es una técnica para determinar la concentración de un elemento de metal particular dentro de una muestra (Aurora Instrument, 2015). Esta técnica se utiliza en gran medida para la cuantificación de casi todos los metales que contienen las muestras provenientes de petróleo (Pascual et al., 2006) y que presenta ventajas como las menciona Razmilic (1994): se determinan más de 60 elementos en un rango de $\mu \mathrm{g} / \mathrm{mL}-\mathrm{ng} / \mathrm{mL}$; es aplicada en una gran variedad de muestras entre los que se pueden mencionar aguas, muestras geológicas muestras orgánicas, metales y aleaciones, petróleo y sus subproductos; muestras químicas y farmacéuticas. Y presenta alta especificidad, sensibilidad y facilidad de operación.

Esta técnica también presenta algunas limitaciones o desventajas como es el sistema quemador del nebulizador, que es un dispositivo de muestreo relativamente ineficiente ya que sólo una pequeña fracción de la muestra llega a la llama y la muestra atomizada pasa rápidamente a través de la trayectoria de la luz, el equipo no se puede dejar sin vigilancia debido a que trabaja con gases inflamables (Perkin Elmer, 2013). Otras desventajas que presenta la técnica son que la absorción es un método de determinación unielemental, es decir se determina un metal a la vez (Rubinson y Rubinson, 2001); la muestra debe ser tratada previamente ya que debe 
estar en estado líquido para tener en la solución orgánica o acuosa, átomos gaseosos libres; puede haber variación en la concentración de átomos del analito libre como resultado de una tasa no uniforme de aspiración, nebulización, atomización y en los pulverizadores de la llama (Harvey, 2000); se pueden presentar interferencias espectrales, físicas, químicas o de ionización en las mediciones (Gallego et al., 2013).

La espectroscopia de absorción atómica presenta también una serie de aspectos que se deben tomar en cuenta durante los análisis, así, por ejemplo, cuando un disolvente orgánico se analiza por espectrometría de absorción atómica de llama, contribuye eficazmente con combustible adicional a la llama, por lo que se recomienda un nebulizador ajustable para la aspiración de disolventes orgánicos; se debe controlar la tasa de absorción dependiendo del disolvente, la cual debe estar alrededor de 1 o $2 \mathrm{~mL} / \mathrm{min}$ (Agilent Technologies, 2015). Los gases que se utilizan son acetileno y óxido nitroso grado UAP y aire, la presión del cilindro de acetileno debe ser superior a $100 \mathrm{psi}(700 \mathrm{kPa})$ para asegurar que la acetona no pueda penetrar en el instrumento (Agilent Technologies, 2015). Se debe controlar las velocidades de combustión porque las llamas sólo son estables en ciertos intervalos de caudal y controlar la afectación de las variaciones de las temperaturas de la llama (Skoog et al., 2001), de acuerdo con el comportamiento de cada metal a medir.

Un requisito de la espectroscopia atómica es que la muestra esté en estado líquido, ya que ésta sería la forma más fácil de manejarla. La mayoría de matrices orgánicas e inorgánicas que se utilizan en análisis elemental requieren la disolución parcial o total de la muestra antes del análisis instrumental. Y esto por lo general implica una preparación simple o más compleja de la muestra. Estos pasos son generalmente la parte más crítica de análisis, ya que son responsables de los errores más importantes (Ramadan, 2011), como no lograr una cantidad suficiente de átomos libres del metal de interés en la llama para la medición de la absorción debido a una incorrecta disociación de las moléculas del analito. Hay varias técnicas para la descomposición de muestras sólidas que implica la liberación del metal de interés a partir de una matriz de interferencia mediante el uso de un reactivo y/o calor. Sin embargo, hay que tener presente que también la utilización de reactivos o ácidos puede causar la contaminación o pérdida de los analitos (Ramadan, 2011). Entre las técnicas de descomposición de muestras sólidas se pueden nombrar: Dilución, digestión ácida (descomposición húmeda o asistida por microondas), incineración o calcinación.

\section{METODOLOGÍA}

\section{Muestras}

Muestra de asfalto codificada por LanammeUCR como M1697-16 proveniente de Moín en Limón y muestreada en Recope el 13 de julio del 2016 del tanque No 7S11.

\section{Materiales y Métodos}

Este método se divide en 4 partes: I Dilución de muestra de asfalto. II Optimización del equipo de absorción atómica. III Medición de concentración de metales en el equipo de AA. IV Confiablidad del método.

\section{Parte. Dilución de Muestra de Asfalto}

El asfalto se calentó en un horno (Despatch, USA) a $135^{\circ} \mathrm{C}$ alrededor de 40 minutos según el procedimiento descrito en ASTM D5/D5M (American Standard for Testing and Materials, 2013). Se agitó con un agitador de vidrio hasta homogenizarlo. En un balón aforado de $50 \mathrm{~mL}$ se pesó en balanza analítica (Ohaus, USA) aproximadamente $0,2500 \mathrm{~g}$ (para medir Zn), 1,000 g (para medir V y Ni) y 1,500 g (para medir Fe). Se tomó la precaución de que no se adhirió el hilo de asfalto en las paredes del balón, ni sobre la marca de aforo. Se dejó enfriar el asfalto por 1 hora. Luego se pesó nuevamente el balón con el asfalto y se determinó el peso exacto de la muestra pesada por diferencia de pesos.

Para la dilución se agregó $20 \mathrm{~mL}$ de mineral spirits CAS $\mathrm{N}^{\circ}$ 8052-41-3 marca Caterpillar y el balón aforado se colocó en un baño ultrasónico por 20 minutos. En caso de que la muestra se caliente se deberá dejar enfriar completamente antes de aforar con mineral spirits, pero de preferencia se debe evitar esto. Es recomendable verificar si la dilución se dió de manera correcta revisando de manera visual si en el balón quedan partículas visibles de asfalto.

\section{Parte. Optimización del Equipo de Absorción Atómica}

Las cuantificaciones de los analitos se realizaron empleando un espectrofotómetro de absorción atómica (EAA) marca Varian modelo AA240FS, serie EL08083339 (Australia, 2004). Este es un equipo de doble haz que utiliza lámparas de cátodo hueco de cada elemento y como corrección de fondo usa la lámpara de deuterio $\left(\mathrm{D}_{2}\right)$. Con ayuda del manual 
de operación del instrumento (Agilent Technologies (2013) y Varian Australia (2000)) se establecieron los parámetros instrumentales en el Software Spectr AA Versión 5.1 PRO de Varian de acuerdo al metal seleccionado. Entre los parámetros importantes debe mencionarse que el instrumento debe determinar absorbancia; se usaron 3 réplicas tanto para estándares como para las muestras con una duración de 5 segundos por lectura con un tiempo de homogenización de 10 segundos para que la muestra ingrese al quemador; con una calibración lineal.

Una vez que fueron ingresadas las condiciones de trabajo de cada de metal al software del equipo de AA se procedió a buscar la mayor absorbancia en cada parámetro en el siguiente orden: condiciones de la lámpara de cátodo hueco, condiciones del quemador y condiciones de la llama.
La Tabla 1 muestra las condiciones óptimas de trabajo para la lámpara de cátodo hueco para cada uno de los metales a determinar y de la lámpara de deuterio. Para lograr esta optimización, se alineó cada lámpara moviendo manualmente los tornillos que las sujetan hasta lograr la máxima absorbancia registrada en el software.

La posición del quemador se verifica con la cartilla de alineación del equipo que se muestra en la Figura 1. El haz de luz de la lámpara se alineó mediante las técnicas recomendadas por el fabricante del equipo (Varian). Se realizaron tres mediciones en la posición horizontal y una medición en la posición vertical. Las mediciones se hicieron con reglas metálicas milimétricas, y se muestran en la Tabla 2.

Tabla 1. Condiciones de la lámpara de cátodo hueco para medir V, Ni, Zn y Fe en muestras de asfalto AC-30 por EAA

\begin{tabular}{|c|c|c|c|c|}
\hline Parámetro & \multicolumn{4}{|c|}{ Medición utilizada } \\
\hline Elemento & $\mathrm{V}$ & $\mathrm{Ni}$ & $\mathrm{Zn}$ & $\mathrm{Fe}$ \\
\hline Corriente $(\mathrm{mA})$ & 20,0 & 4,0 & 5,0 & 5,0 \\
\hline Longitud de onda $(\mathrm{nm})$ & 318,5 & 232,0 & 213,9 & 248,3 \\
\hline Ancho de la rejilla $(\mathrm{nm})$ & 0,2 & 0,2 & 1,0 & 0,2 \\
\hline Corrección de Background $\left(\mathrm{D}_{2}\right)$ & Encendida & Encendida & Encendida & Encendida \\
\hline
\end{tabular}
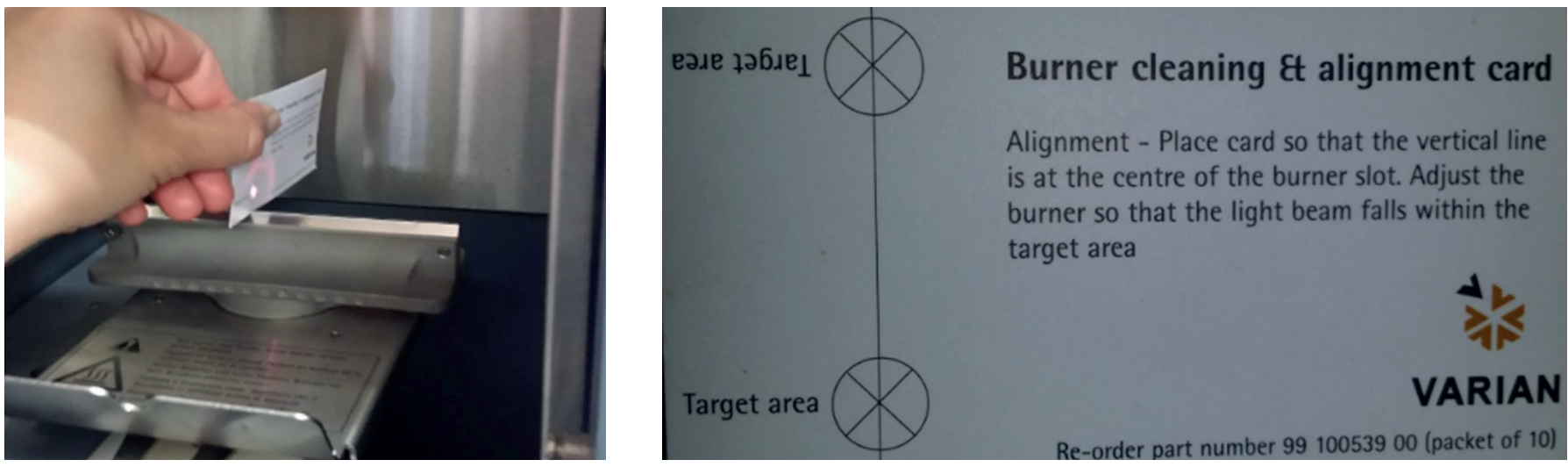

Figura 1. Carta de linealidad del haz de luz para EAA.

Tabla 2. Alineación del quemador de titanio del EAA para medición de $\mathrm{V}$, Ni, Zn y Fe en muestras de asfalto AC-30

\begin{tabular}{|c|c|c|c|c|}
\hline \multicolumn{1}{|c|}{ Parámetro } & \multicolumn{3}{|c|}{ Medición utilizada } \\
\hline Elemento & $\mathrm{V}$ & $\mathrm{Ni}$ & $\mathrm{Zn}$ & 10,0 \\
\hline Tipo de quemador $(\mathrm{cm})$ & 5,0 & 10,0 & 300,0 & 300,0 \\
\hline Posición vertical $(\mathrm{mm})$ & 290,0 & 298,0 & 88,0 & 89,0 \\
\hline Posición horizontal inicial $(\mathrm{mm})$ & 89,0 & 88,5 & 88,0 & 89,0 \\
\hline Posición horizontal media $(\mathrm{mm})$ & 89,0 & 88,5 & 89,5 & 30,0 \\
\hline Posición horizontal final $(\mathrm{mm})$ & 89,5 & 89,0 & 30,0 \\
\hline Tiempo de precalentamiento $(\mathrm{min})$ & 30,0 & 30,0 & 39,0 \\
\hline
\end{tabular}


Para determinar las condiciones de trabajo de la llama para cada uno de los metales en estudio se establecieron los parámetros que se detallan en la Tabla 3 . Se encontró la relación adecuada combustible/oxidante mediante la regulación de los gases, hasta que se alcanzó la máxima absorbancia. Posteriormente mediante el regulador de la bola de impacto se optimizó la nebulización, encontrando la posición donde se obtuvo la mayor absorbancia y flujo adecuado.

\begin{tabular}{|c|c|c|c|c|}
\hline Fe en mu & lestras de a & sfalto AC. & 30 por EAF & 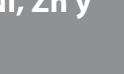 \\
\hline Parámetro & & Mediciór & utilizada & \\
\hline Elemento & V & $\mathrm{Ni}$ & $\mathrm{Zn}$ & $\mathrm{Fe}$ \\
\hline Tipo de llama & $\begin{array}{l}\text { Acetileno - } \\
\text { NOx }\end{array}$ & $\begin{array}{c}\text { Aire - } \\
\text { Acetileno }\end{array}$ & $\begin{array}{c}\text { Aire - } \\
\text { Acetileno }\end{array}$ & $\begin{array}{c}\text { Aire - } \\
\text { Acetileno }\end{array}$ \\
\hline Flujo de aire (L/min) & 11,0 & 13,5 & 13,5 & 13,5 \\
\hline $\begin{array}{l}\begin{array}{l}\text { Flujo de acetileno } \\
\text { (L/min) }\end{array} \\
\end{array}$ & 6,9 & 2,0 & 2,0 & 2,0 \\
\hline Presión de aire (psi) & 40,0 & 50,0 & 50,0 & 50,0 \\
\hline $\begin{array}{l}\text { Presión de acetileno } \\
\text { (psi) }\end{array}$ & 13,0 & 12,0 & 13,0 & 12,0 \\
\hline Largo de capilar (cm) & 26,0 & 26,0 & 26,0 & 26,0 \\
\hline Flujo de capilar (s) & 1,5 & 1,5 & 1,3 & 1,2 \\
\hline
\end{tabular}

\section{Parte. Medición de Concentración de Metales en el Equipo de AA.}

Para la elaboración de la curva de calibración se utilizó un estándar multielemental, V-23 Wear Metal Standard in hydrocarbon oil @ $900 \mu g / g$ de VHG Labs. Las curvas de calibración se realizaron con patrones de concentración conocidas, con un mínimo de 5 puntos, cuyas concentraciones se prepararon a partir de la concentración de trabajo del elemento a determinar. Se prepararon patrones, calculando que la concentración de la muestra quede aproximadamente en la mitad de la curva de calibración. Para construir la gráfica de absorbancia versus concentración, se midieron las absorbancias de las soluciones estándar empezando con un blanco y luego ordenadamente de menor a mayor concentración los estándares, cumpliendo en un rango lineal con la Ley de Lambert y Beer (Fonseca, 2015).

Los patrones se prepararon a partir de un estándar de concentración conocida y diluidos luego en un balón aforado de $50 \mathrm{~mL}$ con mineral spirits. En el caso de V y Ni se usó una alícuota de $25 \mathrm{~mL}$ de solución matriz de asfalto (20 g de asfalto en $1 \mathrm{~L}$ de mineral spirits) para lograr una matriz lo más parecida posible a las muestras y evitar interferencias. En el caso de Zn y Fe no se recomienda usar la matriz de asfalto en los patrones o estándares de calibración ya que las absorbancias son muy altas y la curva resultante no cumple la Ley de Beer. En este caso para el Ni se prepararon estándares de 0,5 a 2,5 ppm, para el V de 5 a 9 ppm, para el Zn de 0,1 a 0,8 ppm y para el Fe de 0,05 a 0,5 ppm.

Posteriormente se verificó la linealidad de la curva mediante el coeficiente de correlación lineal, $\mathrm{R}^{2} \geq 0,995$ mediante hoja de cálculo Excel ${ }^{\circ}$ de Microsoft Office. Una vez elaborada la curva de calibración se ingresó la muestra de asfalto diluida en el capilar del nebulizador del equipo y se procede con la lectura de la absorbancia del metal deseado. El valor de absorbancia se sustituyó por el valor de la ordenada "-y-" en la ecuación de la curva y se despeja el valor de la abscisa "-x-" que corresponde a la concentración real del metal contenido en las muestras de asfalto. Las mediciones de las concentraciones de cada metal se realizaron en 6 muestras de las cuales se determinó la concentración promedio final.

\section{Parte. Confiablidad del Método}

Para determinar la confiabilidad en los métodos desarrollados para la determinación de V, Ni, Zn y Fe en asfalto AC-30 se establecieron criterios como repetibilidad $\mathrm{y}$ exactitud en las mediciones.

Para la repetibilidad en cada metal se pesaron 6 muestras con el peso correspondiente a cada metal a medir y de cada muestra se realizaron 3 réplicas de medición para luego determinar la desviación estándar $(\mathrm{S})$ para un muestral menor de 30 unidades de especímenes y el coeficiente de variación (CV) o desviación estándar relativa (DER) cuyo criterio de aceptación fue de un máximo de $2 \%$ bajo la fórmula:

$\%$ CV o \% DER $=\underset{\text { Pesviación estándar de absorbancias }}{\text { Promedio de la totalidad de absorbancias }}$ "100

Para verificar la exactitud del método se realizó la medición del porcentaje de recuperación, el cual se preparó agregando la cantidad de estándar mutielemental (V-23 Wear Metal Standard in hydrocarbon oil @ $900 \mu \mathrm{g} / \mathrm{g}$ de VHG Labs) necesario en $50 \mathrm{~mL}$, cubriendo el $80 \%$, el $100 \%$ y el $120 \%$ de la concentración aproximada de las muestras en V, Ni y Fe. Se agregó una alícuota de $25 \mathrm{~mL}$ de solución matriz de asfalto a cada estándar y se terminó de aforrar con mineral spirits; para el Zn se usó una alícuota de $10 \mathrm{~mL}$ en cada estándar debido a la alta absorbancia que presentan las muestra en la medición de este metal, ya que se si utiliza una mayor cantidad de solución matriz la curva de calibración tiende a presentar valores de absorbancia mayores a 1 lo cual provoca la caída de la curva de calibración. Cada porcentaje 
de recuperación se trabajó por triplicado. Se determinó en todas las muestras el porcentaje de recuperación mediante la siguiente fórmula:

\% Recuperación $=\underline{\text { Dato experimental }} * 100$ Dato teórico

Como criterio de aceptación, se consideró una recuperación satisfactoria entre $97 \%$ y $103 \%$, es decir de un $\pm 3 \%$ (Vaca, 2013). Sin embargo, para metales que presentan alta absortividad se ha permitido hasta un $12 \%$ como un criterio aceptable (Vega y Vélez, 2011).

\section{RESULTADOS Y DISCUSIÓN}

\section{Parte. Dilución de Muestra de Asfalto}

La dilución de muestras de asfalto con mineral spirits es un procedimiento de bajo costo donde no se presentan tantos riesgos de seguridad durante las pruebas de dilución. Tiene la ventaja que se pueden trabajar pesos de muestras en cantidades menores o igual a 2 g obteniéndose una dilución adecuada. El tiempo de duración es alrededor de 1 hora a 1,5 horas por muestra. Se comprobó de manera visual que no hubiera partículas en la solución, lo cual es de mucha importancia para asegurar la homogenización de la muestra y su correcta lectura en el instrumento.

\section{Parte. Optimización del Equipo de Absorción Atómica}

En la Tabla 4 se detallan las máximas energías finales obtenidas después de la optimización completa del método de cuantificación de V, Ni, Zn y Fe en el equipo de absorción atómica, además muestra las máximas absorbancias alcanzadas en la lámpara de cátodo hueco de cada metal y en la lámpara de Deuterio en cada etapa de optimización. Para alcanzar las energías señaladas en cada etapa de optimización se buscó llegar a la mayor absorbancia en cada etapa de optimización del equipo de AA. El valor de absorbancia máxima lograda entre la lámpara de cada metal y la de $D_{2}$ no puede variar en un valor mayor a 0,1 debido a que esto significa que se introduce mayor rango de error o de diferencia entre las lámparas. La "absorbancia del cero" correspondiente al valor obtenido cuando el equipo lee el solvente o blanco que se usó en cada medición. Para este estudio se tomó como blanco el aire, ya que el valor del solvente usado se tomó para restarlo en las lecturas de las muestras y curvas de calibración, con excepción del Fe donde sí se tomó el valor del mineral spirits como blanco inicial para mejorar la curva de calibración lo que permitió obtener buenas lecturas de absorbancias.

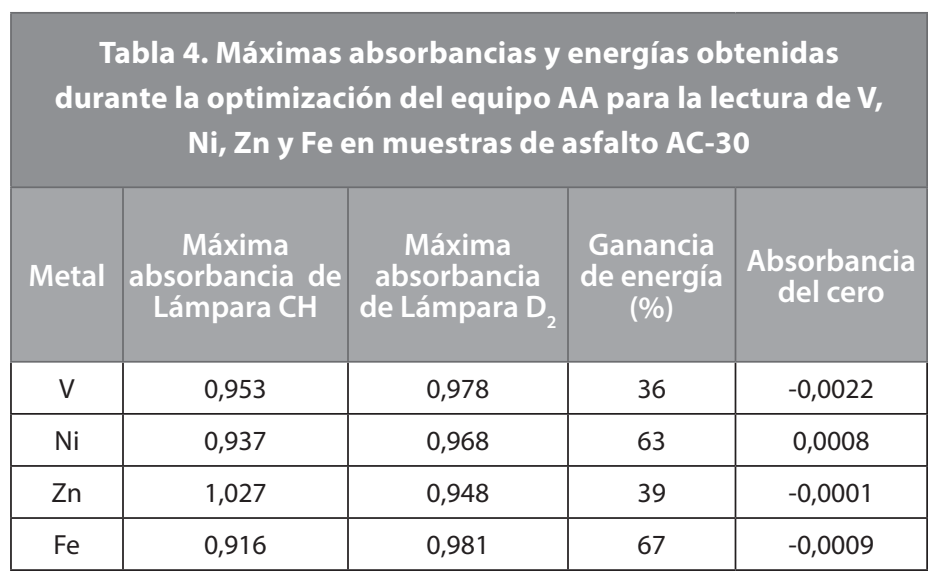

\section{Parte. Medición de Concentración de Metales en el Equipo de $A A$.}

Los resultados de la curva de calibración del Ni dieron como ecuación de linealidad

$\mathrm{X}=(\mathrm{y}-0,0111) / 0,0891$ dando como resultado una concentración de $70 \mathrm{ppm}$ de $\mathrm{Ni}$ contenidas en el asfalto. Para el caso del V, la ecuación fue $\mathrm{X}=(\mathrm{y}-0,0126) / 0,0070$ obteniéndose 330 ppm, mientras que para el Zn, la ecuación de linealidad que se obtuvo fue $\mathrm{X}=(\mathrm{y}-0,2159) / 0,3419$ donde la concentración detectada fue $24 \mathrm{ppm}$. Finalmente, para el Fe la ecuación que se obtuvo fue $\mathrm{X}=(\mathrm{y}-0,0015) /$ 0,1051 y se determinó 10 ppm de Fe en la muestra de asfalto AC-30. Los metales que se encontraron en mayor proporción en la muestra de asfalto AC-30 son el V y Ni, mientras que los que presentan menor concentración son los de Zn y Fe. Los valores del coeficiente de correlación lineal se observan en la Figura 2, para todos los metales, el valor de este parámetro es aceptable, superando el valor límite establecido para $R^{2}=0,995$ y muy cercano a 1 . Esto indica que el grado de relación entre la absorbancia y la concentración es intenso. El Ni fue el metal que mejor $R^{2}$ presentó, mientras que el $R^{2}$ más bajo fue el del $\mathrm{Zn}$, sin embargo, los cuatro valores son aceptables para trabajar con una curva de calibración lineal en un equipo de absorción atómica. 


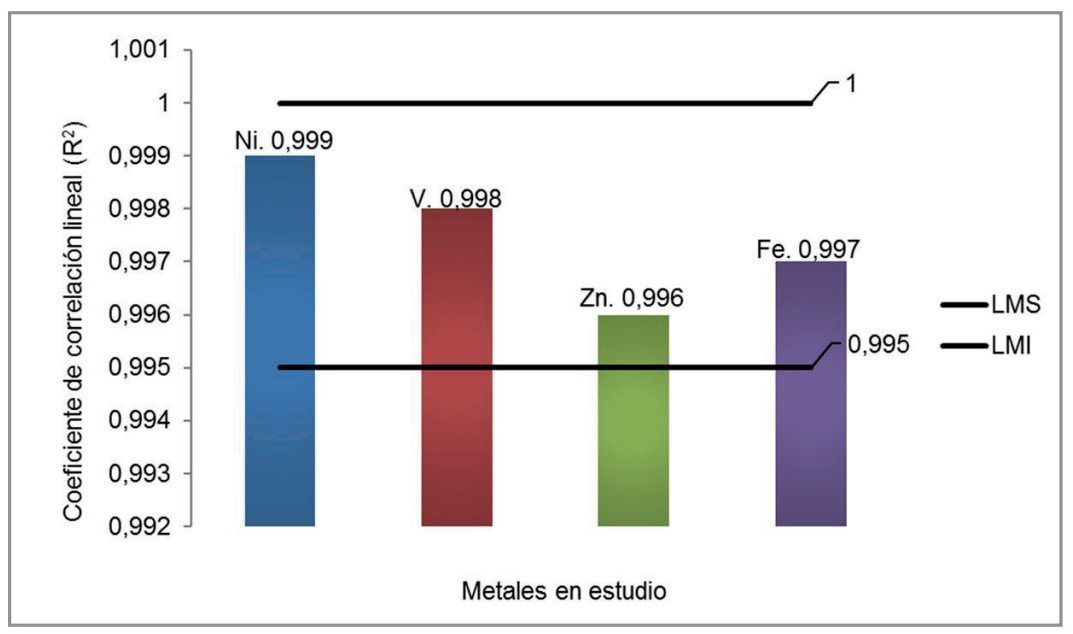

Figura 2. Resultados de coeficiente de correlación lineal $\left(\mathrm{R}^{2}\right)$ para la curva de $\mathrm{Ni}, \mathrm{V}, \mathrm{Zn}$ y Fe en muestras de asfalto $\mathrm{AC}-30$ por EAA.

\section{Parte. Confiablidad del Método}

El cálculo del coeficiente de variación (CV) o desviación estándar relativa (DER) presentó valores menores a $1 \%$ en todos los metales. El Ni presentó un DER de 0,91\%, el V $0,36 \%, \mathrm{Zn} 0,30 \%$ y Fe 0,50\%, estos resultados fueron mejores que los esperados a los indicados como límite aceptable (2\%).

Para determinar la eficiencia del método desarrollado para cada metal se calculó el \% de recuperación de 12 muestras por cada metal, las cuales abarcaron el $80 \%, 100 \%$ y $120 \%$ de la concentración aproximada de la muestra de asfalto según el metal a determinar en el equipo AA, los resultados se pueden observar en la Figura 3, donde se detalla que el $\mathrm{Zn}$ fue el metal que dio resultados con mayor variación ya que incluso para el patrón de recuperación del $80 \%$ su recuperación fue de $90,91 \%$, perdiéndose poco más del $9 \%$ de la medición de Zn en la muestra. De la misma manera se comportaron los resultados para el $100 \%$ y el $120 \%$ en el caso del Zn, mientras que para $\mathrm{Ni}, \mathrm{V}$ y Fe los resultados mostraron una recuperación satisfactoria, donde el porcentaje de recuperación fue inferior al 3\% estimado como límite de aceptación.

Los resultados obtenidos para el Zn y el Fe mostraron $\%$ de recuperación más bajos, se estima pueden ser como resultado de la diferencia en la preparación de muestras y patrones de la curva de calibración, ya que para estos metales no se consideró la matriz de asfalto en los estándares de calibración de la curva. Esto señala que al ser diferente la tensión superficial de la muestra de asfalto para medir Zn y Fe a los patrones o estándares que sólo se prepararon con mineral spirits, se presentan interferencias de matriz que pueden ocurrir en el proceso de nebulización. Ya que, si la muestra y el estándar no son introducidos al sistema de la misma forma, la absorbancia de los átomos no puede correlacionarse entre los dos y producen interferencias de matriz o bien se pueden dar dispersión de la radiación por partículas de la matriz en la muestra que disminuyen la intensidad del haz transmitido, que dan lugar a errores analíticos (Skoog et al., 2001).

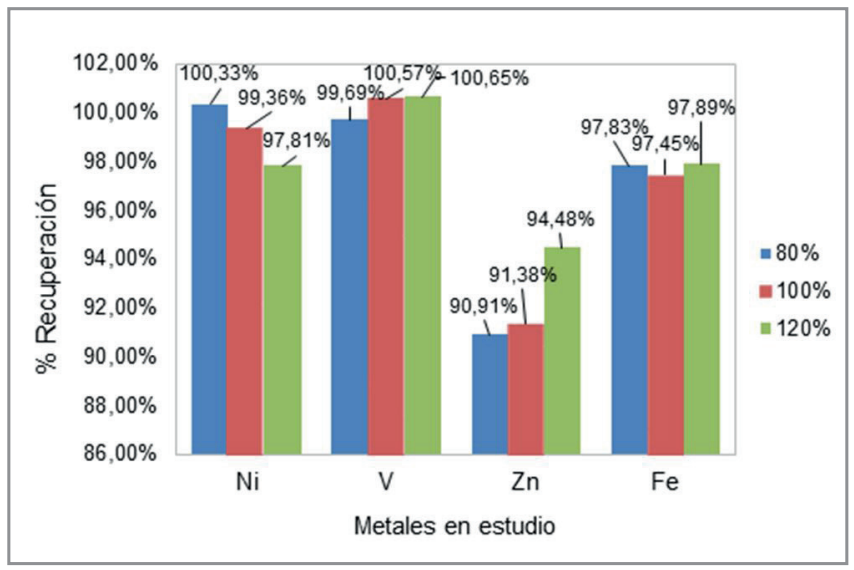

Figura 3. Promedio de los \% recuperación al $80 \%, 100 \%$ y $120 \%$ de la concentración aproximada de $\mathrm{Ni}, \mathrm{V}, \mathrm{Zn}$ y Fe en muestras de asfalto AC-30 por EAA 


\section{CONCLUSIONES}

- Para el tratamiento de muestras de asfalto AC-30 pesadas directamente en balones aforados de boquilla ancha (para evitar que se adhiera el asfalto a las paredes del balón), se determinó que el método por disolución con solvente orgánico (mineral spirits) es un método adecuado ya que no genera ningún riesgo de explosión, no hay exposición a ácidos, no hay riesgo de pérdida de muestra, el procedimiento es rápido, y permite el procesamiento hasta de $2 \mathrm{~g}$ de muestra, lo que permite una dilución adecuada.

- La optimización de parámetros instrumentales en el equipo de AA puede presentar variaciones en absorbancias y energías adquiridas si se cambian las condiciones indicadas en la optimización instrumentales del equipo (alineación de la lámpara de cátodo hueco y la de deuterio, alineación del quemado y regulación de la temperatura de la llama y del flujo usado en los métodos), sin embargo, el cambio de condiciones no implica que el método será lineal o reproducible, sino que puede dar condiciones aceptables para trabajar la determinación de metales mediante EAA.

- La técnica de espectrometría de absorción atómica para medir V, Ni, Zn y Fe en muestras de asfalto, resultó ser un método preciso, ya que los resultados presentan una concordancia aceptable entre las réplicas de la muestra en estudio. Además de que su DER dio resultados inferiores al $1 \%$ lo que indica que las lecturas de la misma muestra no presentaron resultados con errores aleatorios significativos. Es decir, no se presentó una dispersión importante entre los resultados obtenidos mediante esta técnica de EAA.

- Finalmente, la técnica de espectrometría de absorción atómica para medir V, Ni, Zn y Fe en muestras de asfalto también resultó ser un método adecuado para la medición de estos metales, debido a los resultados obtenidos en la determinación del porcentaje de recuperación fueron aceptables para todos los metales en estudio, no superando el 3\% permitido para V, Ni y Fe y el 12\% para Zn.

- Si este método de cuantificación de metales en muestras de asfalto se realiza de forma rutinaria en el Laboratorio de Ligantes de Lanamme, es recomendable someter este método analítico a un proceso de pruebas adecuadas y documentadas, que permitan dar cumplimiento a las especificaciones y atributos de calidad, es decir, realizar un proceso de validación del método a cada uno de los métodos desarrollados para la cuantificación de $\mathrm{V}, \mathrm{Ni}, \mathrm{Zn}$ y Fe determinados mediante absorción atómica en llama, ya que cada uno utiliza un método distinto de detección.

\section{REFERENCIAS BIBLIOGRÁFICAS}

Agilent Technologies Inc. (2013). Agilent 240/280 Series AA (including Zeeman). 13ra ed. Malasia: Autor.

- Agilent Technologies Inc. (2015). Flame Atomic Absorption Spectrometry. Analytical Methods. 13th Ed. Australia Autor.

- American Standard for Testing and Materials. ASTM D5/D5M. (2013). Standard Test Method for Penetration of Bituminous Materials. 4 páginas. Estados Unidos.

- Aurora Instruments Ltd. (2015). Preparation of Petrochemical Samples for Atomic Absorption Spectrometric Analysis. Canadá. Autor.

- Bishara, S. (1997). New, simple, rapid, and precise method for trace analysis of nickel and vanadium in petroleum asphalt. Transportation Research Record: Journal of the Transportation Research Board, (1586), 40-49.

Branthaver, J., Nazir, M., Petersen, J., Dorrence, S. y Ryan, M. (1984). The effect of mattalo porhyrins on asphalt oxidation. II. The effect of vanadylchelates found in petroleum. Liquid Fuels Technology, 2(1), 67-89.

Cárdenas, J. y Fonseca, E. (2009). Modelación del comportamiento reológico de asfalto convencional y modificado con polímero reciclado, estudiada desde la relación viscosidad-temperatura. Revista EIA, 12(2), 125-137.

- Cortés, H. (2012). Análisis del proceso de oxidación del asfalto colombiano 80/100 en inmersión bajo agua periodo 12-21 meses. Magister en Ingeniería Civil. Pontificia Universidad Javeriana

- Fonseca, E. (2015). Aplicación de un método espectrofotométrico de absorción atómica para el análisis de hierro, calcio, magnesio, potasio, y sodio en cemento portland. Licenciado en Ciencias Químicas, con mención en Química Analítica. Pontificia Universidad Católica del Ecuador.

Gallego, A.; Garcinuño, R. y Morcillo, M. (2013). Experimentación en química analitica. Madrid Universidad Nacional de Educación a Distancia.

García, D. y Melgar G. (2011). Validación del método espectrofotométrico (3500-fe D) de la fenantrolina para determinación de hierro total en agua potable. Tesis de Licenciatura en Química y Farmacia. Universidad de El Salvador.

- Harvey, D. (2000). Atomic Absorption Spectroscopy. En Modern Analytical Chemistry (pp. 412-422). USA McGraw-Hill Higher Education.

Nahar, S., Schmets, A., Scarpas, A. y Schmets, A. (2015). Determining Trace Elements in Bitumen by Neutron Activation Analysis. In Transportation Research Board 4th Annual Meeting (No. 15-0321).

- Pascual, J., Hechavarría, J., de Zayas, M., Ferrer, O., Dennes, E. y Álvarez, O. (2006). Determinación de vanadio por espectrometría de absorción atómica en fuel oils. Revista CENIC Ciencias Químicas, 37(1), 8-10.doi ISSN 1015-8553 
Perkin Elmer. (2013). Atomic spectroscopy - A guide to selecting the appropriate technique and system. USA: Autor.

- Ramadan, N. (2011). Sample preparation for flame atomic absorption spectroscopy anverview. Rasāyan. 4(1), 49-55.doi ISSN 0974-1496

Razmilic, B. (1994). Espectroscopia de absorción atómica. En Control de calidad de insumos y dietas acuícolas. México Merck Química Chilena.

Robertson, R., Branthaver, J., Plancher, H., Duvall, J., Ensley, E., Harnsberger, P. y Petersen, J. (1991). Chemical properties of asphalts and their relationship to pavement performance (No. SHRP-A/UWP-91-510). Washington, DC Strategic Highway Research Program, National Research Council.

Rubinson, K y Rubinson, J. (2001). Análisis Instrumental. Madrid Peaeson Educación.

Skoog, D.; Holler, F y Nieman, T. (2001). Principios de Análisis Instrumental. España McGraw-Hill.5ta Ed.

- Speight, J. (2014). The chemistry and technology of petroleum. USA CRC press.
Speight, J. (2016). Asphalt. Materials Science and Technology. USA Butterworth-Heinemann.

Vaca, R. (2013). Validación del método analítico para la determinación de arsénico, mercurio y selenio por espectroscopia de absorción atómica con un sistema de generación de hidruros, en lixiviados con fines de acreditación en el laboratorio de Química Ambiental del OSP. Químico de alimentos. Universidad Central del Ecuador.

Varian Australia Pty. (2000). Atomic Spectroscop y Publications. [CD]. Versión 1.0.

Vega, A y Vélez, P. (2011). Validación del método de determinación de cobre y zinc por espectroscopia de absorción atómica de llama en agua cruda y tratada para el laboratorio de análisis de aguas y alimentos de la Universidad Tecnológica de Pereira. Tecnólogo en Química. Universidad Tecnológica de Pereira. 